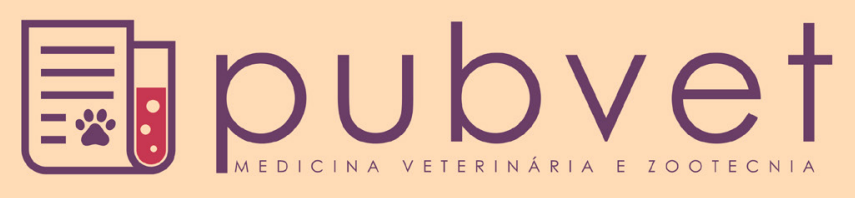

HTTP://DX.DOI.ORG/10.22256/PUBVET.V11N5.460-465

\title{
Amputação da articulação radiocarpal de um pombo doméstico (columba livia): Relato de caso
}

\author{
Ítalo Sena Carvalho ${ }^{*}$, Demétrio Pereira de Sousa Filho ${ }^{1}$, Robson dos Anjos Honorato \\ ${ }^{1}$ Médico Veterinário autônomo, graduado pela Universidade Federal do Piauí-Campus Professora Cinobelina Elvas-Bom Jesus-PI, Brasil. \\ ${ }^{2}$ Médico Veterinário Graduado Pela Universidade Federal Rural de Pernambuco; Especialização em Clínica Médica e Cirúrgica de Cães e \\ Gatos - Faculdades INTA; Residência em Clínica Médica e Cirúrgica de Cães e Gatos - UFPI; Mestrando em Ciência Animal (com ênfase \\ em terapia celular) Pelo Programa de Pós-Graduação da Universidade Federal do Piaui-UFPI. * Autor para correspondência: \\ italo.med.vet@gmail.com.
}

RESUMO. Um pombo doméstico (Columba livia), macho, pesando 200 gramas foi atendido na Clínica Médica Veterinária CMV-SAÚDE (Santa Maria-DF), o qual foi resgatado por um casal de idosos que observaram que o mesmo não conseguia alçar voo. Ao exame físico observou-se fratura exposta na porção distal do III metacarpiano, com processo inflamatório acentuado e alto grau de necrose tecidual. Devido ao grau de necrose tecidual e infecção no local da fratura, não foi possível realizar qualquer técnica de osteossíntese. Sendo assim, a conduta terapêutica adotada foi a amputação baixa na articulação radiocarpal. Tal conduta foi o tratamento ideal para aliviar a dor e reestabelecer o bem-estar do animal.

Palavras chave: aves, fratura de asa, hemograma,

\section{Amputation successful in radiocarpal joint of domestic pigeon (Columba livia): Report a case}

ABSTRACT. A domestic pigeon (Columba livia), male, weighing 200 grams was attended at the CMV-SAÚDE Veterinary Medical Clinic (Santa Maria-DF), which was rescued by an elderly couple who observed that it could not take flight. Physical examination revealed an exposed fracture in the distal portion of the metacarpal III, with a marked inflammatory process and a high degree of tissue necrosis. Due to the degree of tissue necrosis and infection at the fracture site, it was not possible to perform any osteosynthesis technique. Therefore, the therapeutic approach adopted was the low amputation in the radiocarpal joint. Such conduct was the ideal treatment to relieve pain and restore the animal's wellbeing.

Keywords: birds, fracture radiocarpal, blood count

\section{Amputación de la articulación radiocarpiana de un palomo doméstico (Columba livia): Reporte de un caso}

RESUMEN. Un palomo doméstico (Columba livia), con peso de 200 gramos fue atendido en la Clínica Veterinaria CMV-SALUD (Santa Maria-DF), el mismo fue rescatado por una pareja de ancianos que observaron que no podía levantar vuelo. El examen físico reveló una fractura abierta en la porción distal del tercer metacarpiano, con proceso inflamatorio severo y alto grado de necrosis de los tejidos. Debido al grado de necrosis de los tejidos e infección en el sitio de la fractura, no fue posible realizar cualquier técnica de osteosíntesis. Por lo tanto, el enfoque terapéutico adoptado fue amputación de la articulación 
radiocarpiana, siendo este el tratamiento ideal para aliviar el dolor y restaurar el bienestar del animal.

Palabras clave: aves, fractura de ala, hemograma

\section{Introdução}

No cotidiano da clínica de aves existem inúmeras causas para a consulta a um médico veterinário, seja por problemas de origem infecciosa, parasitária ou até mesmo traumática. De acordo (McCartney, 1994), a causa mais comum da consulta veterinária em aves são as fraturas, principalmente dos ossos longos das asas e dos membros pélvicos.

Aves de vida livre estão mais susceptíveis a fraturas de membros, que podem ser ocasionadas por diversos fatores, tais como acidentes com obstáculos impostos pelo homem, os fios de luz ou telefone, fios de cerca, telas, grades, vidros que refletem árvores, armas de fogo ou outras barreiras físicas que impedem o voo livre (Bolson, 2005).

$\mathrm{Na}$ rotina da clínica veterinária, é comum a procura por correções de fraturas traumáticas em aves cativas e de vida livre, mas existem poucos profissionais capacitados para realizar os diversos tipos de técnicas cirúrgicas e tratamentos indicados. Para sucesso do tratamento, é necessário escolher a técnica mais adequada ao caso, priorizando a melhor estabilização e síntese óssea, prevenindo recorrências da fratura ou insucesso terapêutico (Prazeres et al., 2013).

No caso de fraturas expostas, com contaminação severa da ferida, lesões do tipo esmagamento que levam à necrose muscular progressiva e retardo no tempo de vascularização, resultam em amputação (Kafrouni \& Neto, 1987).

O objetivo desse trabalho é descrever um caso de amputação baixa na asa de um pombo doméstico na articulação radiocarpal.

\section{Relato de caso}

Foi atendido na Clínica Médica Veterinária CMV-SAÚDE, Santa Maria-DF, um pombo doméstico (Columba livia), macho, pesando 200 gramas, o qual foi resgatado por um casal de idosos que observaram que o mesmo não conseguia alçar voo devido a uma ferida na asa. Ao exame clínico constatou-se frequência cardíaca de 200 batimentos por minutos e temperatura retal $42,2{ }^{\circ} \mathrm{C}$. Observou-se uma fratura exposta na porção distal do III metacarpiano, com processo inflamatório acentuado e grande quantidade de tecido necrosado (Figura 1). Solicitou-se hemograma completo, cujo resultado está na tabela 1. A conduta terapêutica adotada foi à amputação baixa na articulação radiocarpal.

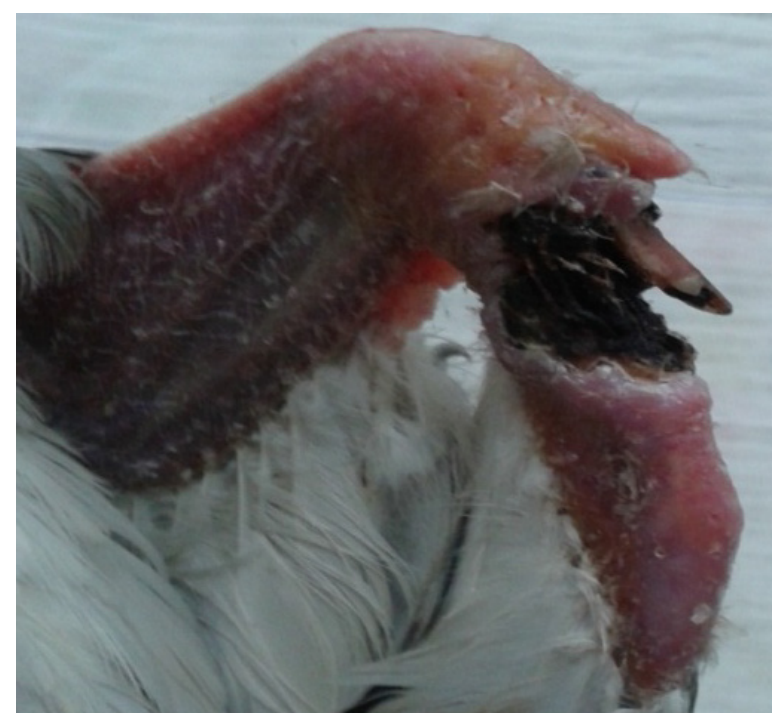

Figura 1. Pombo doméstico (Columba livia) com fratura exposta na porção distal do III metacarpiano. Note a presença de necrose tecidual acentuada. Fonte: Arquivo pessoal.

A estabilização do quadro clínico do paciente antes do procedimento cirúrgico foi realizada administrando-se o cetoprofeno $-5 \mathrm{mg} / \mathrm{kg}$ a cada 12 horas por via oral (VO) amoxicilina com clavulonato de potássio - $125 \mathrm{mg} / \mathrm{kg}$ a cada 12 horas VO; suplemento vitamínico e mineral (adicionada no bebedouro, 15 gotas em $100 \mathrm{~mL}$ de água).

O protocolo anestésico consistiu de tramadol $10 \mathrm{mg} / \mathrm{kg}$ por via intramuscular (IM), midazolam $-2 \mathrm{mg} / \mathrm{kg}$ IM e sulfato de atropina $-0,1 \mathrm{mg} / \mathrm{kg}$ IM como medicação pré-anestésica; cloridrato de ketamina $-50 \mathrm{mg} / \mathrm{kg}$ e xilazina $-5 \mathrm{mg} / \mathrm{kg}$, ambos associados na mesma seringa e aplicados por via intramuscular para indução anestésica. Por se tratar de um procedimento relativamente rápido, não houve necessidade de repetir a dose ou fazer manutenção com anestesia inalatória. Durante o procedimento anestésico-cirúrgico o paciente foi mantido em colchão térmico e com máscara ligada ao cilindro de oxigênio a 100\% para evitar uma possível apneia.

Tabela 1. Valores encontrados no hemograma do pombo doméstico (Columba livia) 


\begin{tabular}{lcc}
\hline Eritrograma & Valores Encontrados & Valores de Referência \\
\hline Hemácias & $2,02 \mathrm{milhões} / \mathrm{mm} 3$ & 2,1 a 4,2 \\
Hemoglobina & $14,30 \mathrm{~g} / \mathrm{dL}$ & 10,7 a 14,9 \\
Hematócrito & $35,00 \%$ & 39,3 a 59,4 \\
VCM & $173,27 \mathrm{fL}$ & 118 a 144 \\
HCM & $70,79 \mathrm{pg}$ & 32 a 48 \\
CHCM & $40,86 \mathrm{~g} / \mathrm{dL}$ & 20 a 30 \\
Trombócitos & $156.000 / \mathrm{mm}^{3}$ & - \\
Leucograma & & 10.000 a 30.000 \\
Leucócitos & $8.800 / \mathrm{mm}^{3}$ & 15 a $50 \%$ \\
Heterófilos & $73 \%(6.424)$ & 25 a $70 \%$ \\
Linfócitos & $26 \%(2.288)$ & 1 a $3 \%$ \\
Monócitos & $1 \%(88)$ & 0 a $1,5 \%$ \\
Eosinófilos & $0 \%(0)$ & 0 a $1 \%$ \\
Basófilos & $0 \%(0)$ & \\
\hline
\end{tabular}

Fonte dos valores de referência: Guimarães (2007).

Com o animal anestesiado e posicionado em decúbito lateral direito, procedeu-se a antissepsia da região e colocação de pano de campo. Realizou-se uma incisão transversal na pele ao redor da porção proximal do III metacarpiano, dissecção do tecido subcutâneo e, em seguida, incisão dos tendões da musculatura local e cápsula articulação radiocarpal. A aproximação dos cotos musculares (Figura 2) foi realizada com fio de nylon 4-0 confeccionando padrão de sutura interrompida em X (sutura de Sutam).

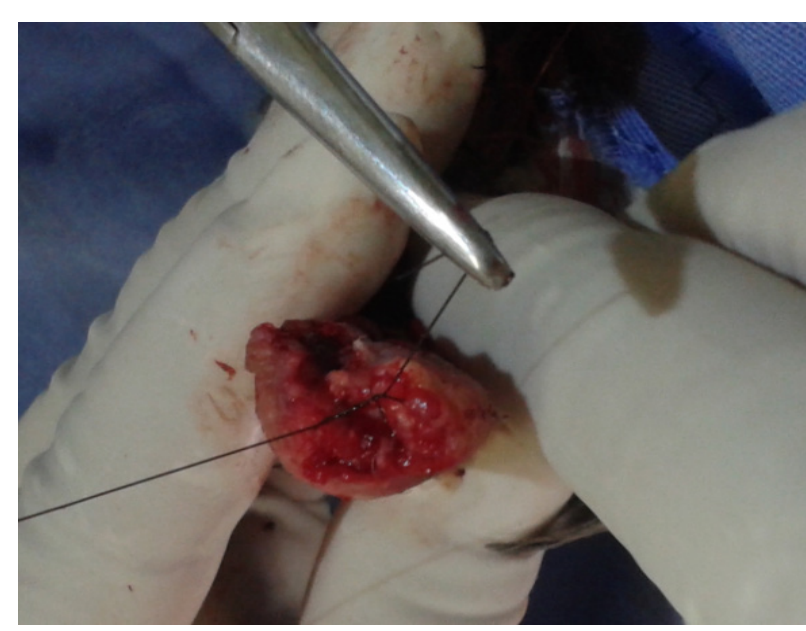

Figura 2. Aproximação da musculatura com sutura de Sutam. Fonte: Arquivo pessoal.

Para dermorrafia utilizou-se o mesmo fio, sendo realizada uma sutura em pontos interrompidos simples (Figura 3).

Após o procedimento cirúrgico fez-se aplicação de pomada antibiótica e cicatrizante e realizou-se curativo com gaze estéril. A terapêutica do pós-operatório consistiu de cetoprofeno $-5 \mathrm{mg} / \mathrm{kg}$ a cada 12 horas (VO), durante cinco dias; amoxicilina com clavulonato de potássio - $25 \mathrm{mg} / \mathrm{kg}$ a cada 12 horas $\mathrm{VO}$, durante sete dias; tramadol $-10 \mathrm{mg} / \mathrm{kg}$ a cada 12 horas durante 5 dias; suplemento vitamínico e mineral (adicionada no bebedouro, 15 gotas em $100 \mathrm{~mL}$ de água); para a ferida cirúrgica, limpeza com gaze e cloreto de sódio $0,9 \%$ e aplicação de pomada antibiótica e cicatrizante a base de sulfato gentamicina, sulfanilamida, sulfadiazina, ureia e vitamina $\mathrm{A}$, duas vezes ao dia até a cicatrização total.

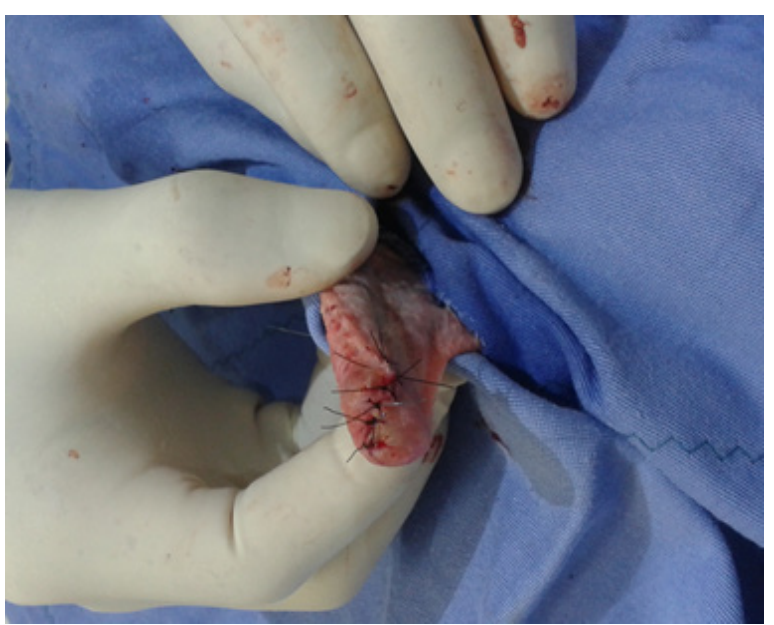

Figura 3. Finalização do procedimento cirúrgico com padrão de sutura interrompida simples. Fonte: Arquivo pessoal.

A retirada dos pontos cirúrgicos foi realizada após 10 dias e a ferida cirúrgica encontrava-se ainda na última fase da cicatrização (remodelagem). Com 35 dias após o procedimento cirúrgico, o processo de cicatrização já havia se completado macroscopicamente e as penas já estavam em fase de crescimento (Figura 4).

\section{Discussão}


Aves submetidas a altos graus de injúria podem sofrer fraturas nos mais diversos ossos e os mais comumente envolvidos, em ordem decrescente, são os ossos dos membros pélvicos, asas e crânio. As fraturas dos membros pélvicos são mais comuns em aves de gaiola ou terrestre, e as lesões de asas ou crânio ocorrem geralmente durante o voo (Wood, 1941 Apud Bolson and Schossler (2008). O animal do presente relato é uma ave de vida livre e sofreu uma fratura na asa, que pode ter sido provocada por trauma em obstáculos impostos pelo homem ou por objetos lançados contra a mesma.

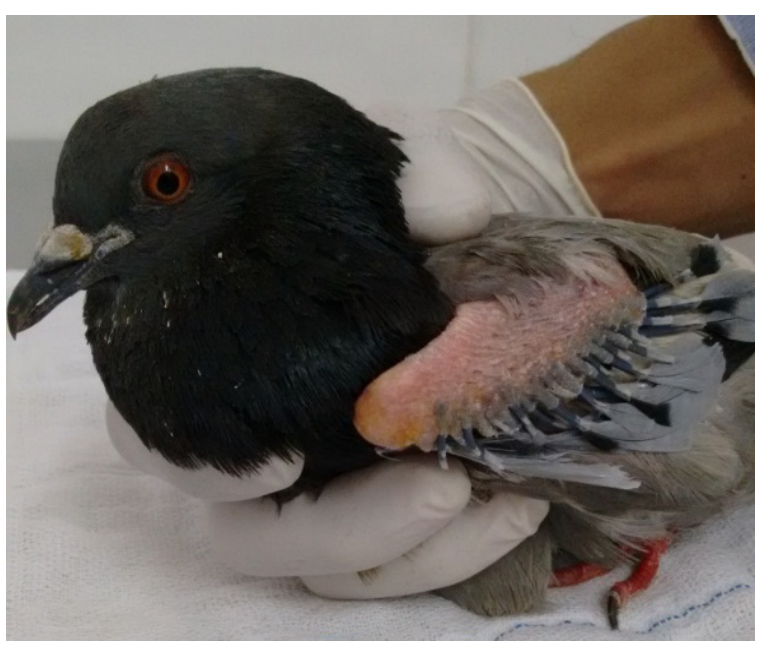

Figura 4. Aspecto do coto da asa do pombo doméstico, 35 dias após a amputação. Macroscopicamente, o processo de remodelação da ferida já está completo e as penas encontramse em crescimento. Fonte: Arquivo pessoal.

Os objetivos do reparo das fraturas em aves são semelhantes aos dos mamíferos, incluindo alinhamento e aposição dos fragmentos ósseos, manutenção da biomecânica da estrutura afetada, obtenção de rígida estabilização após redução e retorno precoce à função (Piermattei and Flo, 2009).

A resolução de uma fratura em uma ave depende do suprimento sanguíneo, ou seja, da integridade muscular adjacente, e da presença ou não de infecção, ocorrendo mais precocemente do que nos mamíferos devido ao alto metabolismo (Miller and Fowler, 1978). Outro fator a ser considerado é o tempo decorrido entre a ocorrência da fratura e a procura ao atendimento médico veterinário.

Pode-se determinar o tratamento e o prognóstico de uma fratura em uma ave por meio de uma classificação precisa da fratura. Sendo que, para chegar a uma classificação exata muitos fatores são considerados. Dentre estes, se a fratura for exposta, tornam-se possíveis infecções ou retardo na resolução, e fraturas que apresentam ferimentos cutâneos profundos associados são classificadas como expostas (Newton and Zeitlin, 1977). Fraturas antigas em membros de aves promovem rapidamente atrofia muscular podendo levar a não união óssea por desuso do membro (Bennett and Kuzma, 1992). Sendo assim nem sempre é possível realizar uma técnica de osteossíntese para todos os casos de fraturas. Alguns casos resultam em amputação. A fratura do paciente do presente relato, além de ser exposta, já se encontrava com alto grau de necrose dos tecidos adjacentes devido à infecção local que se instalou. A amputação baixa na articulação radiocarpal foi a alternativa de tratamento mais adequada. Conduta adotada também por Quadros et al. (2011) que realizaram a amputação parcial de asa em jacu (Penelope obscura) como medida de tratamento para uma fratura exposta oblíqua na porção proximal do rádio e ulna, com dilaceração e necrose dos tecidos adjacentes. Essa mesma conduta foi adotada por Soares et al., (2013) que realizaram a amputação da asa em gavião-carijó (Rupornis magnirostris) com fratura completa exposta em terço proximal de rádio e ulna.

O sucesso ou o infortúnio do ato operatório depende de vários fatores, dentre os quais se destaca a profilaxia da infecção (Costa Neto et al., 2011). O uso apropriado de antimicrobianos na profilaxia cirúrgica é uma das situações que comprovadamente podem exercer um papel importante na prevenção das infecções do sitio cirúrgico. Trata-se de um procedimento recomendado pela boa prática médica (Pereira et al., 2014).

Os antibióticos mais utilizados nas aves são: ciprofloxacino, cloranfenicol, tetraciclina, azitromicina, amoxicilina e ampicilina (Cubas et al., 2007). A antibioticoterapia do paciente em questão foi realizada com amoxicilina associada ao clavulonato de potássio na dose de $125 \mathrm{mg} / \mathrm{kg}$ a cada 12 horas, conforme recomenda Viana (2014). Optou-se por tal medicação por ser uma associação de antibióticos que agem sinergicamente e apresenta um amplo espectro de atividade, atuando contra bactérias Grampositivas e Gram-negativas, inclusive contra cepas produtoras de beta-lactamases.

De extrema importância tal como a antiobioticoterapia em intervenções cirúrgicas, está o controle da dor e do processo inflamatório da ferida cirúrgica. De acordo com Carvalho \& Lemônica, (1998), os mediadores inflamatórios 
uma vez liberados, promovem de forma sinérgica uma alteração no mecanismo de transdução periférica do estímulo nociceptivo, aumentando a sensibilidade de transdução dos nociceptores de elevado limiar, com consequente redução no limiar de percepção do estímulo doloroso, exagerada resposta a estímulos nociceptivos supralimiares (hiperalgesia) e dor espontânea (alodínia). Para Escobar \& Stumm (2016), dentre as inúmeras condições que podem interferir no aumento do tempo de internação de um paciente, está a dor.

Para a recuperação cirúrgica mais rápida de um paciente, é imprescindível o controle da dor e inflamação. Sendo que tal controle pode ser feito utilizando-se somente anti-inflamatório com ação analgésica ou associado ao uso simultâneo de um analgésico, na dependência da natureza da intervenção cirúrgica, se é pouco ou mais invasiva, se envolve somente tecidos moles ou é um procedimento ortopédico. De acordo com Ekman and Koman (2004) procedimentos ortopédicos induzem dor mais intensa do que outros procedimentos cirúrgicos, pois a injúria ao osso é mais dolorosa do que a injúria a tecidos moles, uma vez que o periósteo possui menor limiar de dor somática do que outras estruturas.

Médicos e médicos veterinários devem se conscientizar de que a dor é um fator biológico ativo, que pode causar todas as consequências perniciosas do estresse, como imunossupressão, retardo da cicatrização de feridas, aceleração de processos patológicos, entre outros, e, portanto, tem o dever moral e ético de reconhecer e tratar a dor no homem e nos animais (Fantoni et al., 2002).

$\mathrm{Na}$ prática ortopédica, os opióides são comumente utilizados para tratar a dor moderada a intensa, geralmente aguda, provocada por fraturas e lesões de tecidos moles (Ekman and Koman, 2004). Os anti-inflamatórios não esteroidais (AINES) constituem o grupo de antiinflamatórios mais utilizados para controle da dor leve moderada em medicina humana e veterinária (Andrade, 2008).

Para o controle da dor e inflamação do paciente do presente estudo houve a necessidade de se associar um agente analgésico opióide (tramadol) a um AINE (cetoprofeno), visto que a amputação é um procedimento cirúrgico ortopédico invasivo que provoca dor intensa. Essa associação permitiu o controle da dor e inflamação contribuindo para uma recuperação adequada do paciente.

\section{Conclusão}

A técnica de amputação baixa de asa na articulação radiocarpal do paciente presente relato, foi a conduta mais adequada para tratamento da fratura exposta do III metacarpiano, aliviando a dor e reestabelecendo o bem-estar do mesmo. A antibioticoterapia com amoxicilina associada ao clavulonato de potássio mostrou-se eficaz como terapia antimicrobiana profilática e no período pós-operatório.

\section{Referências Bibliográficas}

Bennett, R. A. \& Kuzma, A. B. 1992. Fracture management in birds. Journal of Zoo and Wildlife Medicine, 23, 5-38.

Bolson, J. 2005. Comportamento do polímero de mamona (Ricinnus communis) em ossos de codornas domésticas (Coturnix japonica). 2005. 57f. Programa de Pós-graduação em Medicina Veterinária. Universidade Federal de Santa Maria, Santa Maria.

Bolson, J. \& Schossler, J. E. W. 2008. Osteossíntese em aves-revisão da literatura. Arquivos de Ciências Veterinárias e Zoologia da UNIPAR, 11, 55-62.

Carvalho, w. A \& Lemônica, L. 1998. Mecanismos Celulares e Moleculares da Dor Inflamatória. Modulação Periférica e Avanços Terapêuticos- Artigo de. Revista Brasileira de Anestesiologia, 48, 137-158.

Costa Neto, J. M., Martins Filho, E. F., Junior, D. C. G., Teixeira, D. M. \& Jesus Moraes, V. 2011. Princípios da assépsia cirúrgica veterinária Roca, São Paulo.

Cubas, Z. S., Silva, J. C. R. \& Dias, J. L. C. 2007. Tratado de animais selvagens-medicina veterinária. Editora Roca.

Ekman, E. F. \& Koman, L. A. 2004. Acute pain following musculoskeletal injuries and orthopaedic surgery. The Journal of Bone and Joint Surgery, 86, 1316-1327.

Escobar, P. \& Stumm, E. M. F. 2016. Avaliação da Dor de pacientes em Pós-operatório Imediato de Cirurgia Traumatológica Relatório técnico-científico. XXIV Seminário de Iniciação Científica. 6 p. Disponível em http://www.publicacoeseventos.unijui.edu.br/i ndexphp/salaoconhecimento/article/viewFile/ 6468/5243.

Fantoni, D. T., Mastrocinque, S., Fantoni, D. T. \& Cottopassi, S. R. 2002. Fisiopatologia e 
controle da dor. In: Fabntoni, D. T. (ed.) Anestesia em cães e gatos. Roca, São Paulo.

Kafrouni M. L.; Neto A. F. Revista do Hospital de Pronto Socorro da Secretaria Municipal de Saúde e Serviço Social, 33, 2, 1987.

McCartney, W. T. 1994. Orthopaedic injuries in pigeons. The Veterinary Record, 134, 305-307.

Miller, R. E. \& Fowler, M. E. 1978. Fowler's Zoo and Wild Animal Medicine Current Therapy.

Newton, C. D. \& Zeitlin, S. 1977. Avian fracture healing. Journal of the American Veterinary Medical Association, 170, 620-625.

Pereira, G. C. B., Lima, L. S., Pinheiro, P. d. N. Q. \& Dolabela, M. F. 2014. Perfil de uso de antimicrobianos em procedimentos de otorrinolaringologia. Revista Para Médica, 28, 31-39.

Piermattei, B. D. L. \& Flo, G. L. 2009. Ortopedia e tratamento das fraturas dos pequenos animais, 3 ed. edn. Editora Manolo, São Paulo.

Prazeres, R. F., Fiebig, W. J., Fecchio, R. S., Biasi, C., Fernandes, M., Souza Castro, M. A. G. \& Pachaly, J. R. 2013. Técnicas de reconstituição de bico em aves-artigo de revisão. Journal of Health Science Institute, 31, 441-447.
Quadros, A. M. et al., Amputação Parcial de Asa em Jacu (Penelope obscura) - Relato de Caso. Congresso brasileiro de medicina veterinária (Combravet). Revista de Ciências Agroveterinárias. 3 p. Florianópolis-SC. 2011. Disponível em: http://www.sovergs.com.br/site/38conbravet/li sta area $02 . h t m$.

Soares, E. S.; Moraes, L. C.; Neto, J. M.; Cypriano, L.; Neves, C. D. Amputação de Asa de Gavião-Carijó (Rupornis Magnirostris): Relato de Caso. XL Seminário Capixaba do Médico Veterinário. I Seminário de Reprodução de Equídeo. p. 5, 2013.

Viana, F. A. B. 2014. Guia terapêutico veterinário.

\section{Article History:}

Received 13 December 2016

Accepted 17 January 2017

Available on line 28 March 2017

License information: This is an open-access article distributed under the terms of the Creative Commons Attribution License 4.0, which permits unrestricted use, distribution, and reproduction in any medium, provided the original work is properly cited. 